\title{
A Spatial and Spectral Analysis of the Emission from the Supernova Remnant G266.2-1.2
}

\section{Glenn E. Allen*}

MIT Kavli Institute, Cambridge, MA, USA

E-mail: gea@space.mit.edu

\section{Tracey DeLaney}

West Virginia Wesleyan College, Buckhannon, WV, USA

E-mail: delaney_t@wvwc.edu

\section{Miroslav Filipović}

University of Western Sydney, Penrith, NSW, Australia

E-mail: m.filipovic@uws.edu.au

\section{John C. Houck}

MIT Kavli Institute, Cambridge, MA, USA

E-mail: houck@space.mit.edu

\section{Thomas G. Pannuti}

Morehead State University, Morehead, KY, USA

E-mail: t.pannuti@moreheadstate.edu

\section{Michael D. Stage}

University of Massachusetts, Amherst, MA, USA

E-mail: mikstage@nova.astro.umass.edu

G266.2-1.2 is a recently discovered supernova remnant observed to produce faint radio and Xray emission as well as $\mathrm{TeV}$ gamma rays. The age, distance, and gamma-ray emission mechanism have been a source of controversy. To help determine the age and distance, we present the results of an analysis of our 2003 and 2008 Chandra observations of the northwestern region. These results show that the remnant is expanding at the rate of $0.43 \pm 0.09 \operatorname{arcsec~}_{\mathrm{yr}^{-1}}$ from which the age and distance are inferred to be $2-9 \mathrm{kyr}$ and $1-3 \mathrm{kpc}$, respectively. Furthermore, the ambient density is inferred to be less than $0.1 \mathrm{~cm}^{-3}$, perhaps much less. To help determine whether the $\mathrm{TeV}$ gamma rays are produced by electrons or protons, we present the results of a joint spectral analysis of some radio, X-ray, and gamma-ray data. It is possible to fit the broad-band data with a simple, single-zone, leptonic model. However, if the $\mathrm{TeV}$ emission is dominated by the decay of neutral pions, then very-high-energy protons must outnumber very-high-energy electrons by a ratio that is much larger than the ratio observed locally (i.e. the ratio expected from charge conservation).

The Extreme and Variable High Energy Sky

September 19-23, 2011

Chia Laguna (Cagliari), Italy

\footnotetext{
${ }^{*}$ Speaker.
} 


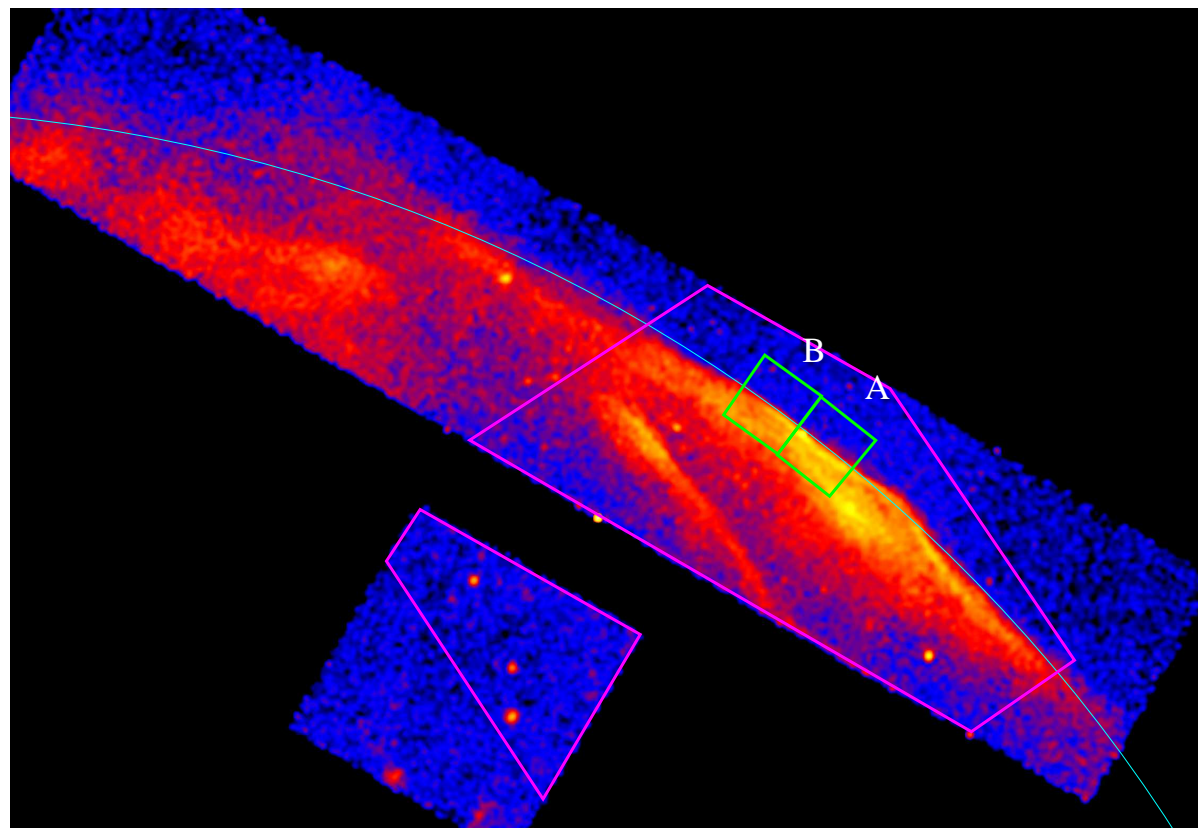

Figure 1: A 1-5 keV image of the northwestern region of G266.2-1.2 from the 2003 Chandra observation. The magenta lines mark the boundary of the region that was observed in both 2003 and 2008. The cyan arc is a segment of a $0.8642^{\circ}$-radius circle centered on the location of a potential central compact object [14]. The green lines mark the boundaries of the regions (A and $\mathrm{B}$ ) used to measure the rate of expansion.

\section{Introduction}

The shell-type supernova remnant G266.2-1.2 (RX J0852.0-4622, "Vela Junior") was discovered in the ROSAT all-sky survey data [1]. It has a diameter of nearly $2^{\circ}$ and was probably the result of a core-collapse event. Like SN 1006, the radio emission is relatively weak [2, 3, 4] and the X-ray emission from the rims is dominated by synchrotron radiation from electrons accelerated at the forward shock $[5,6,7]$. The presence of very-high-energy particles is confirmed by the detection of $\mathrm{TeV}$ gamma-ray emission $[8,9]$. However, the mechanism responsible for this emission-inverse Compton scattering or the decay of neutral pions-remains controversial.

The age and distance of G266.2-1.2 are also a source of controversy. Some argue that the spectrum of the remnant possesses line features associated with the decay of ${ }^{44} \mathrm{Ti}[7,10,11]$. In this case, the remnant must be young $(0.6-1.2 \mathrm{kyr})$ and close $(0.1-0.5 \mathrm{kpc})$ or the line emission would be undetectable [12]. Others find little or no evidence of such features and argue that the relatively large absorption column density indicates that the remnant lies beyond Vela and may be associated with the Vela Molecular Ridge (i.e. at a distance of 1-2 kpc) [5, 6].

We used the Chandra telescope to observe the thin filaments in the bright northwestern region of G266.2-1.2 on two separate occasions. An analysis of these data is used to measure the rate of expansion in this region, which helps determine the age and distance of the remnant. A joint spectral analysis of some ATCA radio [4], Chandra X-ray, and HESS gamma-ray [9] data is performed to investigate whether the gamma rays are produced by electrons or protons. Section 2 describes 


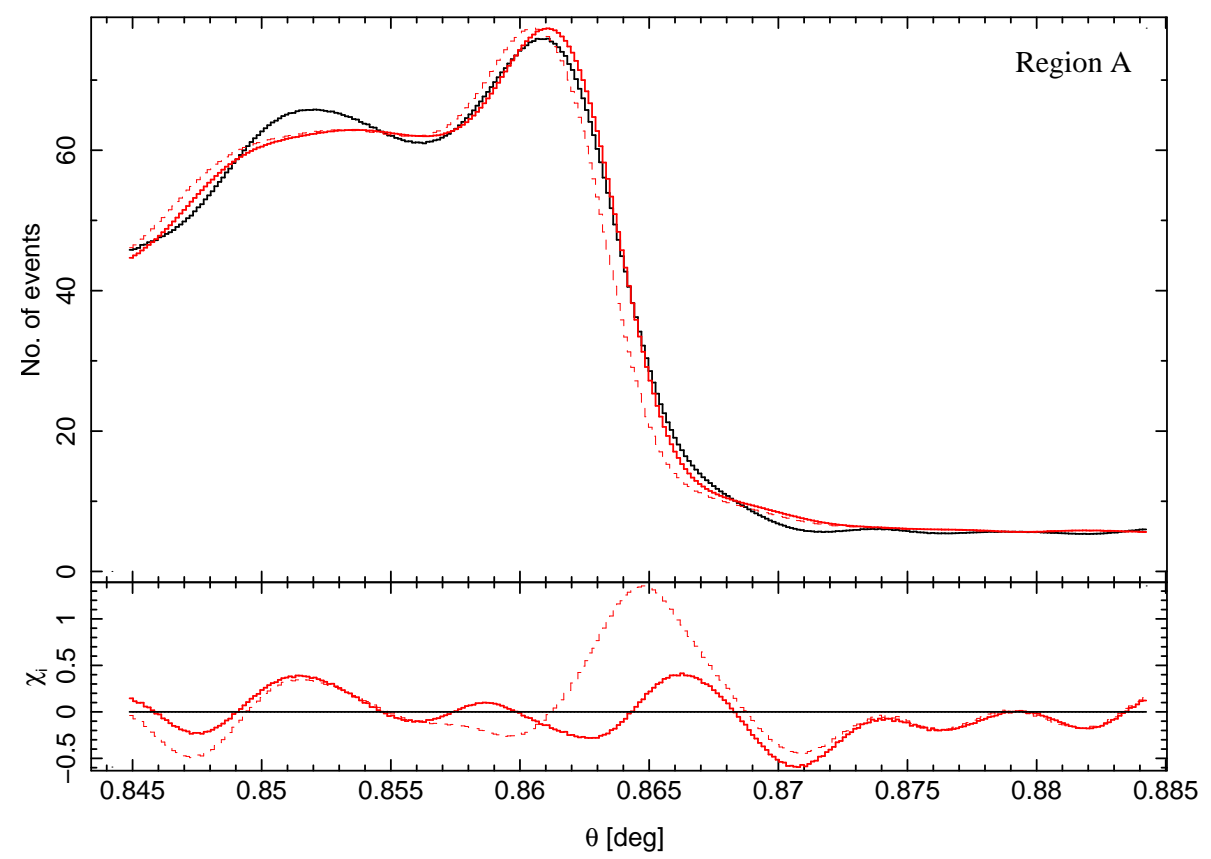

Figure 2: Tom panel: Radial profiles of region "A" (see Fig. 1) for the 2008 data (black curve), the unshifted 2003 data (dashed red curve), and the shifted (by $2^{\prime \prime}$ ) 2003 data (solid red curve). Bottom panel: The differences between the red profiles and the black profile divided by the $1 \sigma$ statistical uncertainties.

the Chandra data and the analyses. The results of the analyses are discussed in section 3.

\section{Data and Analyses}

We observed the northwestern region of G266.2-1.2 with Chandra in 2003 [13] and 2008. Both sets of ACIS data were reprocessed in the usual manner with CIAO 4.1. The data at energies less than $1 \mathrm{keV}$ were discarded because they are dominated by emission from the Vela remnant. The data at energies greater than or equal to $5 \mathrm{keV}$ were discarded because they are dominated by a charged-particle background. An image of the remaining 2003 data is displayed in Figure 1.

After confirming that the relative registration of the 2003 and 2008 data is accurate to about 0.5 " ( 1 pixel) or better, radial profiles were obtained for annular wedges " $A$ " and " $B$ " in Figure 1. The amount of the radial expansion in each annular wedge was measured by (1) shifting the 2003 data by a small amount $\Delta \theta$ in the radial direction, (2) smoothing the shifted data with a two-dimensional Gaussian ( $\sigma_{x}=\sigma_{y}=4.92^{\prime \prime}=10$ pixels, which is comparable to the point-spread function of the telescope along much of the outer rim), (3) constructing a one-dimensional radial profile for the shifted and smoothed data (bin width $=0.492^{\prime \prime}=1$ pixel), (4) adjusting this profile to compensate for differences in the number of background events, in the observation times, and in the detection efficiency with respect to the 2008 data, (5) smoothing the 2008 data with the same two-dimensional Gaussian, (6) constructing a one-dimensional radial profile for the 2008 data, and (7) calculating the value of $\chi^{2}$ (i.e. the sum of the squares of the residuals in the lower panel of Figure 2). Many iterations of this fitting process were performed using different values for the 


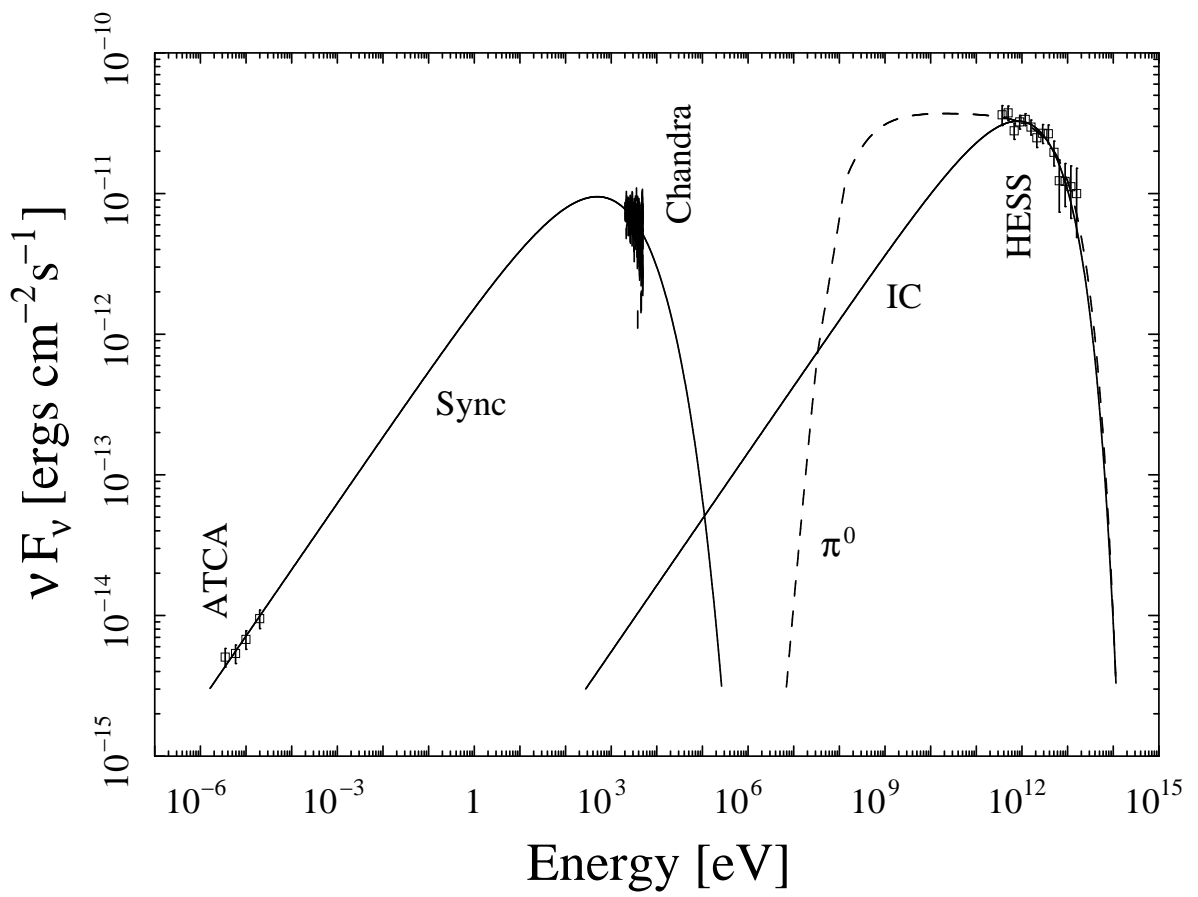

Figure 3: The radio to gamma-ray photon energy-flux spectrum of G266.2-1.2. The data, which are labeled vertically, include the ATCA radio [4] and 2008 Chandra X-ray data for part of the northwestern region, and the HESS data [9] for the entire remnant. The three model spectra are the fitted results for the emission produced by synchrotron radiation (Sync), inverse Compton scattering of the cosmic microwave background radiation (IC), and the decay of neutral pions $\left(\pi^{0}\right)$.

Table 1. Expansion rate

\begin{tabular}{ccccc}
\hline \hline Region & $\begin{array}{c}\text { Shift } \\
{[\operatorname{arcsec}]}\end{array}$ & $\begin{array}{c}\text { Expansion rate } \\
{\left[\operatorname{arcsec} \mathrm{yr}^{-1}\right]}\end{array}$ & $\begin{array}{c}\text { Radius } \\
{[\mathrm{deg}]}\end{array}$ & $\begin{array}{c}\text { Expansion rate } \\
{\left[\% \mathrm{yr}^{-1}\right]}\end{array}$ \\
\hline $\mathrm{A}$ & $2.0 \pm 0.5$ & $0.35 \pm 0.09$ & 0.8642 & $0.011 \pm 0.003$ \\
$\mathrm{~B}$ & $3.0 \pm 0.6$ & $0.53 \pm 0.11$ & 0.8648 & $0.017 \pm 0.003$ \\
\hline
\end{tabular}

amount of the radial shift and for the relative normalizations of the 2003 and 2008 profiles. The results for region A are shown in Figure 2. Note that the location of a potential compact central object [14] is used as the location of the center of the remnant. The results for the expansion rate do not vary significantly for centers within $0.2^{\circ}$ of this location.

A joint spectral analysis of the ATCA radio [4], Chandra X-ray, and HESS gamma-ray [9] data was performed using the spectral-fitting package ISIS [15] and the synchrotron, inverse Compton scattering, and neutral-pion decay models of [16] (Fig. 3). The electron and proton particle spectra are assumed to be exponentially cut-off power laws.

\section{Discussion}

A statistically significant expansion is found for regions A and B (Table 1). The weighted 
Table 2. Hydrodynamical parameters

\begin{tabular}{lcc}
\hline \hline Parameter & $m=0.4$ & $m=1$ \\
\hline$t[\mathrm{kyr}]$ & 2.9 & 7.2 \\
$E_{0}[\mathrm{erg}]$ & $\left(10^{51}\right)$ & $\left(10^{51}\right)$ \\
$n_{0}\left[\mathrm{~cm}^{-3}\right]$ & $\left(10^{-2}\right)$ & $\left(10^{-3}\right)$ \\
$r[\mathrm{pc}]$ & 19 & 37 \\
$d[\mathrm{kpc}]$ & 1.3 & 2.5 \\
$v\left[10^{3} \mathrm{~km} \mathrm{~s}^{-1}\right]$ & 2.6 & $(5.0)$ \\
$M_{\mathrm{s}}\left[M_{\odot}\right]$ & 10 & 7.4 \\
\hline
\end{tabular}

The values in parentheses are assumed values.

Table 3. Spectral parameters

\begin{tabular}{lccc}
\hline \hline Parameter & $\begin{array}{c}\text { Inverse } \\
\text { Compton }\end{array}$ & $\begin{array}{c}\text { Neutral pion decay } \\
\text { with } E_{\text {max }, \mathrm{e}}=E_{\text {max }, \mathrm{p}}\end{array}$ & $\begin{array}{c}\text { Neutral pion decay } \\
\text { with } B=100 \mu \mathrm{G}\end{array}$ \\
\hline$\Gamma_{\mathrm{e}}$ & $2.06_{-0.04}^{+0.03}$ & $2.05_{-0.04}^{+0.03}$ & $2.05_{-0.04}^{+0.03}$ \\
$\Gamma_{\mathrm{p}}$ & $\ldots$ & $=\Gamma_{\mathrm{e}}$ & $=\Gamma_{\mathrm{e}}$ \\
$E_{\text {max }, \mathrm{e}}[\mathrm{TeV}]$ & $17.9_{-2.8}^{+3.6}$ & $=E_{\max , \mathrm{p}}$ & $11.1_{-1.4}^{+1.8}$ \\
$E_{\text {max,p }}[\mathrm{TeV}]$ & $\ldots$ & $89_{-26}^{+45}$ & $89_{-26}^{+45}$ \\
$B[\mu \mathrm{G}]$ & $39_{-13}^{+21}$ & $1.6_{-0.8}^{+1.5}$ & $100(\mathrm{fixed})$ \\
$K_{\mathrm{ep}}$ & $\ldots$ & $8.4 \times 10^{-4}\left(\frac{V_{\mathrm{p}}}{V_{B}}\right)\left(\frac{n_{0}}{10^{-2} \mathrm{~cm}^{-3}}\right)$ & $1 \times 10^{-6}\left(\frac{V_{\mathrm{p}}}{V_{B}}\right)\left(\frac{n_{0}}{10^{-2} \mathrm{~cm}^{-3}}\right)$ \\
\hline
\end{tabular}

mean for these two regions is $\dot{\theta}=0.43 \pm 0.09 \operatorname{arcsec} \mathrm{yr}^{-1}$. For comparison, [17] report a $5.5 \operatorname{arcsec}$ expansion over a $6.5 \mathrm{yr}$ interval from 2001 to 2007 (i.e. $0.85 \pm 0.22 \operatorname{arcsec}_{\mathrm{yr}^{-1}}$ ). If the angular radius $\theta=r / d$, where $d$ is the distance, the radius $r=a t^{m}, a$ is a constant, and the expansion parameter $m$ is the current shock velocity $v$ divided by the mean shock velocity over time, then the age $t=m \theta / \dot{\theta}$. It is unlikely that $m<0.4$ because the presence of thin, X-ray-synchrotrondominated filaments and the lack of thermal X-ray emission [5] suggest that G266.2-1.2 has not evolved beyond the Sedov phase. If $m=0.4$, then $t=2.9 \pm 0.7 \mathrm{kyr}$. Since this result represents a lower limit on the age, the remnant is too old to produce an observable flux of photons from the decay of ${ }^{44} \mathrm{Ti}$. Furthermore, if the initial kinetic energy $E_{0}=10^{51} \mathrm{erg}$ and the ambient density $n_{0}=10^{-2} \mathrm{~cm}^{-3}$, then self-similar considerations [18] yield the other parameters in the second column of Table 2. While $n_{0}$ could be somewhat larger than $10^{-2} \mathrm{~cm}^{-3}$, it seems unlikely that it could be much larger than $0.1 \mathrm{~cm}^{-3}$. Otherwise the remnant might have swept up enough material $\left(M_{\mathrm{s}}\right)$ to produce an observable flux of thermal X-ray emission.

At the other extreme if $m=1$, then G266.2-1.2 is in the free-expansion phase and $t=7.2 \pm$ $1.7 \mathrm{kyr}$. Furthermore, if $v=5000 \mathrm{~km} \mathrm{~s}^{-1}$ and $n_{0}=10^{-3} \mathrm{~cm}^{-3}$, then the other parameters in the third column of Table 2 are obtained. If $m=1, n_{0}$ must be quite low. Otherwise, the remnant would have swept-up too much material to be in the free-expansion phase.

As shown in Figure 3, it is possible to fit the gamma-ray data using models dominated by either inverse Compton scattering of the cosmic microwave background or the decay of neutral 
pions. The differential spectral indices $\left(\Gamma_{\mathrm{e}}\right.$ and $\left.\Gamma_{\mathrm{p}}\right)$, exponential cut-off energies $\left(E_{\max , \mathrm{e}}\right.$ and $\left.E_{\mathrm{max}, \mathrm{p}}\right)$, magnetic field strength $(B)$, and ratio of the number density of very-high-energy electrons to the number density of very-high-energy protons $\left(K_{\mathrm{ep}}\right)$ are listed in Table 3 . These parameters and the ratio of the volume filled by the very-high-energy electrons to the volume filled by the amplified magnetic field $\left(V_{\mathrm{e}} / V_{B} \sim 5\right)$ seem reasonable for the inverse Compton model. Some might think that the magnetic field strength $\left(39_{-13}^{+21} \mu \mathrm{G}\right)$ is a little low, but bear in mind that in our single-zone model $B$ is a flux-weighted mean. The field strength in the regions with the largest field could be significantly larger.

If the gamma-ray emission is dominated by the decay of neutral pions and if $E_{\max , \mathrm{e}}=E_{\max , \mathrm{p}}$, then $B$ seems implausibly low $\left(1.6_{-0.8}^{+1.5} \mu \mathrm{G}\right.$, which is comparable to or less than typical interstellar values in the Galaxy [19]). Alternatively, if $B=100 \mu \mathrm{G}$ and $E_{\mathrm{max}, \mathrm{e}}$ is a free parameter, then $K_{\mathrm{ep}}$ seems rather low. While $K_{\mathrm{ep}}$ is sensitive to the assumed ambient density and the ratio of the volumes filled by the protons $\left(V_{\mathrm{p}}\right)$ and magnetic field $\left(V_{B}\right)$, even if $V_{\mathrm{p}}=10 V_{B}$ and $n_{0}=0.1 \mathrm{~cm}^{-3}, K_{\text {ep }}$ remains well below the value measured for cosmic rays near Earth $\left(\sim 10^{-2}\right.$, which is consistent with the value expected for charge conservation). Therefore, if $K_{\text {ep }} \ll 10^{-2}$, then G266.2-1.2 has a charge gradient in the neighborhood of the shock. It is not clear that such a gradient can be achieved.

\section{References}

[1] Aschenbach, B., 1998, Nature, 396, 141

[2] Combi, J. A., Romero, G. E. \& Benaglia, P., 1999, ApJL, 519, L177

[3] Duncan, A. R. \& Green, D. A., 2000, A\&A, 364, 732

[4] Stupar, M., et al., 2005, Adv. Space Res., 35, 1047

[5] Slane, P., et al., 2001, ApJ, 548, 814

[6] Bamba, A., Yamazaki, R. \& Hiraga, J. S., 2005a, ApJ, 632, 294

[7] Iyudin, A. F., et al., 2005, A\&A, 429, 225

[8] Katagiri, H., et al., 2005, ApJL, 619, L163

[9] Aharonian, F., et al. 2007, ApJ, 661, 236

[10] Iyudin, A. F., et al., 1998, Nature, 396, 142

[11] Tsunemi, H., et al., 2000, PASJ, 52, 887

[12] Chen, W. \& Gehrels, N., 1999, ApJL, 514, L103

[13] Pannuti, T. G., et al., 2010, ApJ, 721, 1492

[14] Pavlov, G. G., et al. 2001, ApJL, 559, L131

[15] Houck, J. C., Denicola, L. A. 2000, Astronomical Data Analysis Software and Systems IX, ASP Conference Proceedings, ed. Manset, N., et al., 216, 591

[16] Houck, J. C., \& Allen, G. E., 2006, ApJS, 167, 26

[17] Katsuda, S., Tsunemi, H., \& Mori, K., 2008, ApJL, 678, L35

[18] Truelove, J. K., McKee, C. F., 1999, ApJS, 120, 299

[19] Sun, X. H., et al. 2008, A\&A, 477, 573 